\title{
Well-posedness of fractional parabolic equations
}

\section{Allaberen Ashyralyev*}

\section{"Correspondence:}

aashyr@fatih.edu.tr

Department of Mathematics, Fatih

University, Istanbul, 34500, Turkey

\begin{abstract}
In the present paper, we consider the abstract Cauchy problem for the fractional differential equation

$$
\frac{d u(t)}{d t}+D_{t}^{\frac{1}{2}} u(t)+A(t) u(t)=f(t), \quad 0<t<1, \quad u(0)=0
$$

in an arbitrary Banach space $E$ with the strongly positive operators $A(t)$. The well-posedness of this problem in spaces of smooth functions is established. The coercive stability estimates for the solution of problems for $2 m$ th order multidimensional fractional parabolic equations and one-dimensional fractional parabolic equations with nonlocal boundary conditions in a space variable are obtained. The stable difference scheme for the approximate solution of this problem is presented. The well-posedness of the difference scheme in difference analogues of spaces of smooth functions is established. In practice, the coercive stability estimates for the solution of difference schemes for the fractional parabolic equation with nonlocal boundary conditions in a space variable and the $2 m$ th order multidimensional fractional parabolic equation are obtained.
\end{abstract}

MSC: $65 \mathrm{M} 12 ; 65 \mathrm{~N} 12$

Keywords: fractional parabolic equation; Basset problem; well-posedness; coercive stability

\section{Introduction}

It is known that differential equations involving derivatives of noninteger order have shown to be adequate models for various physical phenomena in areas like rheology, damping laws, diffusion processes, etc. Methods of solutions of problems for fractional differential equations have been studied extensively by many researchers (see, e.g., [1-43] and the references given therein).

The role played by coercive stability inequalities (well-posedness) in the study of boundary value problems for parabolic partial differential equations is well known (see, e.g., [4451]). In the present paper, the initial value problem

$$
\frac{d u(t)}{d t}+D_{t}^{\frac{1}{2}} u(t)+A(t) u(t)=f(t), \quad 0<t<1, \quad u(0)=0
$$

for the fractional differential equation in an arbitrary Banach space $E$ with the linear (unbounded) operators $A(t)$ is considered. Here $u(t)$ and $f(t)$ are the unknown and the given

C 2013 Ashyralyev; licensee Springer. This is an Open Access article distributed under the terms of the Creative Commons Attribution License (http://creativecommons.org/licenses/by/2.0), which permits unrestricted use, distribution, and reproduction in any medium, provided the original work is properly cited. 
functions, respectively, defined on $[0, T]$ with values in $E$. The derivative $u^{\prime}(t)$ is understood as the limit in the norm of $E$ of the corresponding ratio of differences. $A(t)$ is a given closed linear operator in $E$ with the domain $D(A(t))=D$, independent of $t$ and dense in $E$. Finally, $u(0)=0$.

Here $D_{t}^{\frac{1}{2}}=D_{0+}^{\frac{1}{2}}$ is the standard Riemann-Liouville derivative of order $\frac{1}{2}$. This fractional differential equation corresponds to the Basset problem [9]. It represents a classical problem in fluid dynamics where the unsteady motion of a particle accelerates in a viscous fluid due to the gravity of force. Recently, fractional Basset equations with independent in $t$ operator coefficients $A(t)=A$ have been studied extensively (see, e.g., [52-56] and the references given therein).

In the present paper, the well-posedness of problem (2) with dependent in $t$ operator coefficients $A(t)$ in spaces of smooth functions is established. In practice, the coercive stability estimates for the solution of problems for $2 m$ th order multidimensional fractional parabolic equations and one-dimensional fractional parabolic equations with nonlocal boundary conditions in a space variable are obtained. The stable difference scheme for the approximate solution of initial value problem (2)

$$
\left\{\begin{array}{l}
\tau^{-1}\left(u_{k}-u_{k-1}\right)+A_{k} u_{k}+\frac{1}{\sqrt{\pi}} \sum_{m=1}^{k} \frac{\Gamma\left(k-m+\frac{1}{2}\right)}{(k-m) !} \frac{u_{m}-u_{m-1}}{\tau^{\frac{1}{2}}}=f_{k}, \\
f_{k}=f\left(t_{k}\right), \quad A_{k}=A\left(t_{k}\right), \quad t_{k}=k \tau, \quad 1 \leq k \leq N, \quad N \tau=1, \quad u_{0}=0
\end{array}\right.
$$

is presented. Here $\Gamma\left(k-m+\frac{1}{2}\right)=\int_{0}^{\infty} t^{k-m-\frac{1}{2}} e^{-t} d t$.

The paper is organized as follows. The well-posedness of problem (2) in spaces of smooth functions is established in Section 2. In Section 3 the coercive stability estimates for the solution of problems for $2 m$ th order multidimensional fractional parabolic equations and one-dimensional fractional parabolic equations with nonlocal boundary conditions are obtained. The well-posedness of (3) in difference analogues of spaces of smooth functions is established and the coercive stability estimates for the solution of difference schemes for the fractional parabolic equation with nonlocal boundary conditions in a space variable and the $2 m$ th order multidimensional fractional parabolic equation are obtained in Section 4.

\section{The well-posedness of problem (2)}

A function $u(t)$ is called a solution of problem (2) if the following conditions are satisfied:

(i) $u(t)$ is continuously differentiable on the segment $[0,1]$. The derivatives at the endpoints of the segment are understood as the appropriate unilateral derivatives.

(ii) The element $u(t)$ belongs to $D(A(t))$ for all $t \in[0,1]$ and the function $A(t) u(t)$ is continuous on the segment $[0,1]$.

(iii) $u(t)$ satisfies the equation and the initial condition (2).

A solution of problem (2) defined in this manner will from now on be referred to as a solution of problem (2) in the space $C(E)=C([0,1], E)$ of all continuous functions $\varphi(t)$ defined on $[0,1]$ with values in $E$ equipped with the norm

$$
\|\varphi\|_{C(E)}=\max _{0 \leq t \leq 1}\|\varphi(t)\|_{E} .
$$


In this paper, positive constants, which can differ in time, are indicated with an $M$. On the other hand, $M(\alpha, \beta, \ldots)$ is used to focus on the fact that the constant depends only on $\alpha, \beta, \ldots$

The well-posedness in $C(E)$ of boundary value problem (2) means that the coercive inequality

$$
\left\|u^{\prime}\right\|_{C(E)}+\|A(\cdot) u\|_{C(E)} \leq M\|f\|_{C(E)}
$$

is true for its solution $u(t) \in C(E)$.

Suppose that for each $t \in[0,1]$ the operator $-A(t)$ generates an analytic semigroup $\exp \{-s A(t)\}(s \geq 0)$ with an exponentially decreasing norm, when $s \rightarrow+\infty$, i.e., the following estimates

$$
\|\exp (-s A(t))\|_{E \rightarrow E},\|s A(t) \exp (-s A(t))\|_{E \rightarrow E} \leq M e^{-\delta s} \quad(s>0)
$$

hold for some $M \in[1,+\infty), \delta \in(0,+\infty)$. From this inequality it follows the operator $A^{-1}(t)$ exists and is bounded, and hence $A(t)$ is closed in $C(E)$.

Suppose that the operator $A(t) A^{-1}(s)$ is Hölder continuous in $t$ in the uniform operator topology for each fixed $s$, that is,

$$
\left\|[A(t)-A(\tau)] A^{-1}(s)\right\|_{E \rightarrow E} \leq M|t-\tau|^{\varepsilon}, \quad 0<\varepsilon \leq 1,0 \leq t, s, \tau \leq 1
$$

An operator-valued function $v(t, s)$, defined and strongly continuous jointly in $t, s$ for $0 \leq s<t \leq 1$, is called a fundamental solution of (2) if

(1) the operator $v(t, s)$ is strongly continuous in $t$ and $s$ for $0 \leq s<t \leq T$,

(2) the following identity holds:

$$
v(t, s)=v(t, \tau) v(\tau, s), v(t, t)=I \quad \text { for } 0 \leq s \leq \tau \leq t \leq 1,
$$

(3) the operator $v(t, s)$ maps the region $D$ into itself. The operator $u(t, s)=A(t) v(t, s) A^{-1}(s)$ is bounded and strongly continuous in $t$ and $s$ for $0 \leq s<t \leq 1$,

(4) on the region $D$ the operator $v(t, s)$ is strongly differentiable relative to $t$ and $s$, while

$$
\frac{\partial v(t, s)}{\partial t}=-A(t) v(t, s)
$$

and

$$
\frac{\partial v(t, s)}{\partial s}=v(t, s) A(s)
$$

Now, let us obtain the representation for the solution of problem (2). The initial value problem

$$
\frac{d u}{d t}+A(t) u(t)=F(t), \quad 0<t<1, \quad u(0)=u_{0}
$$


has a unique solution [54] and the following formula holds:

$$
u(t)=v(t, 0) u_{0}+\int_{0}^{t} v(t, s) F(s) d s
$$

Using $u(0)=0$ and the formula $F(s)=f(s)-D_{s}^{\frac{1}{2}} u(s)$, we get

$$
u(t)=-\int_{0}^{t} v(t, s) D_{s}^{\frac{1}{2}} u(s) d s+\int_{0}^{t} v(t, s) f(s) d s
$$

Now, we will give a series of interesting lemmas and estimates concerning the fundamental solution $v(t, s)$ of (2) which will be useful in the sequel.

Lemma 2.1 For any $0 \leq s<t \leq 1$ and $u \in D$, the following identities hold:

$$
\begin{aligned}
v(t, s) u= & \exp \{-(t-s) A(s)\} u \\
& +\int_{s}^{t} v(t, z)[A(s)-A(z)] A^{-1}(s) \exp \{-(z-s) A(s)\} A(s) u d z, \\
v(t, s) u= & \exp \{-(t-s) A(t)\} u \\
& +\int_{s}^{t} \exp \{-(t-z) A(t)\}[A(z)-A(t)] v(z, s) u d z .
\end{aligned}
$$

Lemma 2.2 For any $0 \leq s<t \leq t+r \leq 1,0 \leq \alpha \leq 1$ and $0 \leq \varepsilon \leq 1$, the following estimates hold:

$$
\begin{aligned}
& \|v(t, s)\|_{E \rightarrow E} \leq M, \\
& \left\|A(t) v(t, s) A^{-1}(s)\right\|_{E \rightarrow E} \leq M, \\
& \|v(t, s)-\exp \{-(t-s) A(t)\}\|_{E \rightarrow E} \leq M(t-s)^{\varepsilon}, \\
& \|A(t)[v(t, s)-\exp \{-(t-s) A(t)\}]\|_{E \rightarrow E} \leq M(t-s)^{\varepsilon-1}, \\
& \|A(t) v(t, s)\|_{E \rightarrow E} \leq M(t-s)^{-1} .
\end{aligned}
$$

Theorem 2.1 Let $A(t)$ be a strongly positive operator in a Banach space E and $f(t) \in C(E)$. Then for the solution $u(t)$ in $C(E)$ of initial value problem (2), the following stability inequality holds:

$$
\left\|D_{t}^{\frac{1}{2}} u\right\|_{C(E)}+\left\|u^{\prime}+A(\cdot) u\right\|_{C(E)} \leq M\|f\|_{C(E)} .
$$

Proof Using formula (12), we get

$$
u^{\prime}(t)=-D_{t}^{\frac{1}{2}} u(t)+f(t)+\int_{0}^{t} A(t) v(t, s) D_{s}^{\frac{1}{2}} u(s) d s-\int_{0}^{t} A(t) v(t, s) f(s) d s .
$$

Applying formula (23) and the formula

$$
D_{t}^{\frac{1}{2}} u(t)=\int_{0}^{t} \frac{u^{\prime}(p) d p}{\sqrt{\pi}(t-p)^{\frac{1}{2}}}
$$


we obtain

$$
\begin{aligned}
D_{t}^{\frac{1}{2}} u(t)= & \int_{0}^{t} \frac{1}{\sqrt{\pi}(t-s)^{\frac{1}{2}}}\left(-D_{s}^{\frac{1}{2}} u(s)+f(s)\right) d s \\
& +\int_{0}^{t} \int_{s}^{t} \frac{1}{\sqrt{\pi}(t-p)^{\frac{1}{2}}} A(p) v(p, s) d p D_{s}^{\frac{1}{2}} u(s) d s \\
& -\int_{0}^{t} \int_{s}^{t} \frac{1}{\sqrt{\pi}(t-p)^{\frac{1}{2}}} A(p) v(p, s) d p f(s) d s .
\end{aligned}
$$

Let us first obtain the estimate

$$
\left\|\int_{s}^{t} \frac{1}{\sqrt{\pi}(t-p)^{\frac{1}{2}}} A(p) v(p, s) d p\right\|_{E \rightarrow E} \leq \frac{M}{\sqrt{t-s}}
$$

for any $0 \leq s<t \leq 1$. We have that

$$
\begin{aligned}
\int_{s}^{t} \frac{1}{\sqrt{\pi}(t-p)^{\frac{1}{2}}} A(p) v(p, s) d p= & \int_{\frac{t+s}{2}}^{t} \frac{1}{\sqrt{\pi}(t-p)^{\frac{1}{2}}} A(p) v(p, s) d p \\
& +\int_{s}^{\frac{t+s}{2}} \frac{1}{\sqrt{\pi}(t-p)^{\frac{1}{2}}} A(p) v(p, s) d p=J_{1}+J_{2}
\end{aligned}
$$

Applying estimate (21), we get

$$
\left\|J_{1}\right\|_{E \rightarrow E} \leq M \int_{\frac{t+s}{2}}^{t} \frac{1}{\sqrt{\pi}(t-p)^{\frac{1}{2}}} \frac{1}{p-s} d p \leq \frac{2 M}{t-s} \int_{\frac{t+s}{2}}^{t} \frac{1}{\sqrt{\pi}(t-p)^{\frac{1}{2}}} d p=\frac{M_{1}}{\sqrt{t-s}} .
$$

Now, we will estimate $J_{2}$. We have that

$$
J_{2}=\frac{1}{\sqrt{\pi} \sqrt{t-s}} I-v\left(\frac{t+s}{2}, s\right) \frac{\sqrt{2}}{\sqrt{\pi} \sqrt{t-s}}+\int_{s}^{\frac{t+s}{2}} \frac{\frac{1}{2}}{\sqrt{\pi}(t-p)^{\frac{3}{2}}} v(p, s) d p
$$

Applying estimate (17), we get

$$
\left\|J_{2}\right\|_{E \rightarrow E} \leq \frac{1}{\sqrt{t-s}}+M \frac{\sqrt{2}}{\sqrt{t-s}}+M \int_{s}^{\frac{t+s}{2}} \frac{1}{2 \sqrt{\pi}(t-p)^{\frac{3}{2}}} d p \leq \frac{M_{2}}{\sqrt{t-s}}
$$

Estimate (26) follows from estimates (29) and (31).

Now, let us first estimate $z(t)=\left\|D_{t}^{\frac{1}{2}} u(t)\right\|_{E}$. Applying the triangle inequality and estimate (26), we get

$$
z(t) \leq M \int_{0}^{t} \frac{1}{\sqrt{t-s}} z(s) d s+M \int_{0}^{t} \frac{1}{\sqrt{t-s}}\|f(s)\|_{E} d s
$$

for any $t \in[0,1]$. Applying the above inequality and the integral inequality, we obtain

$$
\max _{0 \leq t \leq 1} z(t) \leq M \max _{0 \leq t \leq 1}\|f(t)\|_{E}
$$


Using the triangle inequality and equation (2), we get

$$
\begin{aligned}
\max _{0 \leq t \leq 1}\left\|u_{t}+A(t) u(t)\right\|_{E} & \leq\left[\max _{0 \leq t \leq 1}\|f(t)\|_{E}+\max _{0 \leq t \leq 1}\left\|D_{t}^{\frac{1}{2}} u(t)\right\|_{E}\right] \\
& \leq M_{1} \max _{0 \leq t \leq 1}\|f(t)\|_{E} .
\end{aligned}
$$

Estimate (22) follows from estimates (33) and (35). Theorem 2.1 is proved.

With the help of $A(t)$, we introduce the fractional spaces $E_{\alpha}(E, A(t)), 0<\alpha<1$, consisting of all $v \in E$ for which the following norms are finite:

$$
\|v\|_{E_{\alpha}}=\sup _{z>0} z^{1-\alpha}\|A(t) \exp \{-z A(t)\} v\|_{E}
$$

From (6) and (7) it follows that

Theorem 2.2 $E_{\alpha}(E, A(t))=E_{\alpha}(E, A(0))$ for all $0<\alpha<1$ and $0 \leq t \leq 1$.

Problem (2) is not well posed in $C(E)$ for arbitrary $E$. It turns out that a Banach space $E$ can be restricted to a Banach space $E^{\prime}$ in such a manner that the restricted problem (2) in $E^{\prime}$ will be well posed in $C\left(E^{\prime}\right)$. The role of $E^{\prime}$ will be played here by the fractional spaces $E_{\alpha}=E_{\alpha}(A(t), E)(0<\alpha<1)$.

Theorem 2.3 Suppose $f(t) \in C\left(E_{\alpha}\right)(0<\alpha<1)$. Suppose that assumptions (6) and (7) hold and $0<\alpha \leq \varepsilon<1$. Then for the solution $u(t)$ in $C\left(E_{\alpha}\right)$ of problem (2), the coercive inequality

$$
\left\|u^{\prime}\right\|_{C\left(E_{\alpha}\right)}+\|A(\cdot) u\|_{C\left(E_{\alpha}\right)} \leq M \alpha^{-1}(1-\alpha)^{-1}\|f\|_{C\left(E_{\alpha}\right)}
$$

holds.

Proof By Theorem 2.1,

$$
\left\|D_{t}^{\frac{1}{2}} u\right\|_{C\left(E_{\alpha}\right)} \leq M\|f\|_{C\left(E_{\alpha}\right)}
$$

for the solution of initial value problem (2). The proof of the estimate

$$
\|A(\cdot) u\|_{C\left(E_{\alpha}\right)} \leq M \alpha^{-1}(1-\alpha)^{-1}\|f\|_{C\left(E_{\alpha}\right)}
$$

for the solution of initial value problem (2) is based on formula (12), estimate (38) and the following estimates [54]:

$$
\begin{aligned}
& \max _{0 \leq t \leq 1}\left\|\int_{0}^{t} A(t) v(t, s) f(s) d s\right\|_{E_{\alpha}} \leq M \alpha^{-1}(1-\alpha)^{-1}\|f\|_{C\left(E_{\alpha}\right)}, \\
& \max _{0 \leq t \leq 1}\left\|\int_{0}^{t} A(t) v(t, s) D_{s}^{\frac{1}{2}} u(s) d s\right\|_{E_{\alpha}} \leq M \alpha^{-1}(1-\alpha)^{-1}\left\|D_{t}^{\frac{1}{2}} u\right\|_{C\left(E_{\alpha}\right)} .
\end{aligned}
$$


Using equation (2) and the triangle inequality, we get

$$
\begin{aligned}
\max _{0 \leq t \leq 1}\left\|u^{\prime}(t)\right\|_{E_{\alpha}} & \leq\left[\max _{0 \leq t \leq 1}\|f(t)\|_{E_{\alpha}}+\max _{0 \leq t \leq 1}\|A(t) u(t)\|_{E_{\alpha}}+\max _{0 \leq t \leq 1}\left\|D_{t}^{\frac{1}{2}} u(t)\right\|_{E_{\alpha}}\right] \\
& \leq M_{1} \alpha^{-1}(1-\alpha)^{-1} \max _{0 \leq t \leq 1}\|f(t)\|_{E_{\alpha}} .
\end{aligned}
$$

Estimate (37) follows from estimates (39) and (42). Theorem 2.3 is proved.

Let us give, without proof, the following result.

Theorem 2.4 Suppose that assumption (6) holds. Suppose that the operator $A(t) A^{-1}(s)$ is Hölder continuous in $t$ in the uniform operator topology for each fixed s, that is,

$$
\left\|[A(t)-A(\tau)] A^{-1}(s)\right\|_{E_{\alpha} \rightarrow E \alpha} \leq M|t-\tau|^{\varepsilon}, \quad 0<\varepsilon \leq 1,
$$

where $M$ and $\varepsilon$ are positive constants independent of $t$, $s$ and $\tau$ for $0 \leq t, s, \tau \leq T$. Suppose $f(t) \in C\left(E_{\alpha}\right)(0<\alpha<1)$. Then for the solution $u(t)$ in $C\left(E_{\alpha}\right)$ of problem (2), the coercive inequality

$$
\left\|u^{\prime}\right\|_{C\left(E_{\alpha}\right)}+\|A(\cdot) u\|_{C\left(E_{\alpha}\right)} \leq M \alpha^{-1}(1-\alpha)^{-1}\|f\|_{C\left(E_{\alpha}\right)}
$$

holds.

\section{Applications}

Now, we consider the applications of Theorems 2.1, 2.3 and 2.4.

First, the Cauchy problem on the range $\left\{0 \leq t \leq 1, x \in \mathbb{R}^{n}\right\}$ for the $2 m$-order multidimensional fractional parabolic equation is considered:

$$
\left\{\begin{array}{l}
\frac{\partial v(t, x)}{\partial t}+D_{t}^{\frac{1}{2}} v(t, x)+\sum_{|r|=2 m} a_{r}(t, x) \frac{\partial|r| v(t, x)}{\partial x_{1}^{r_{1} \ldots \partial x_{n}^{r_{n}}}}+\sigma v(t, x)=f(t, x), \quad 0<t<1, \\
v(0, x)=0, \quad x \in \mathbb{R}^{n}, \quad|r|=r_{1}+\cdots+r_{n},
\end{array}\right.
$$

where $a_{r}(t, x)$ and $f(t, x)$ are given as sufficiently smooth functions. Here, $\sigma$ is a sufficiently large positive constant.

Let us consider a differential operator with constant coefficients of the form

$$
B=\sum_{|r|=2 m} b_{r} \frac{\partial^{r_{1}+\cdots+r_{n}}}{\partial_{x_{1}^{r_{1}} \cdots \partial_{x_{n}}^{r_{n}}}}
$$

acting on functions defined on the entire space $\mathbb{R}^{n}$. Here $r \in \mathbb{R}^{n}$ is a vector with nonnegative integer components, $|r|=r_{1}+\cdots+r_{n}$. If $\varphi(y)\left(y=\left(y_{1}, \ldots, y_{n}\right) \in \mathbb{R}^{n}\right)$ is an infinitely differentiable function that decays at infinity together with all its derivatives, then by means of the Fourier transformation, one establishes the equality

$$
F\left(B_{\varphi}\right)(\xi)=B(\xi) F(\varphi)(\xi)
$$


Here the Fourier transform operator is defined by the following rule:

$$
\begin{aligned}
& F(\varphi)(\xi)=(2 \pi)^{-n / 2} \int_{\mathbb{R}^{n}} \exp \{-i(y, \xi)\} \varphi(y) d y, \\
& (y, \xi)=y_{1} \xi_{1}+\cdots+y_{n} \xi_{n} .
\end{aligned}
$$

The function $B(\xi)$ is called the symbol of the operator $B$ and is given by

$$
B(\xi)=\sum_{|r|=2 m} b_{r}\left(i \xi_{1}\right)^{r_{1}} \cdots\left(i \xi_{n}\right)^{r_{n}}
$$

We will assume that the symbol

$$
B^{t, x}(\xi)=\sum_{|r|=2 m} a_{r}(t, x)\left(i \xi_{1}\right)^{r_{1}} \cdots\left(i \xi_{n}\right)^{r_{n}}, \quad \xi=\left(\xi_{1}, \ldots, \xi_{n}\right) \in \mathbb{R}^{n}
$$

of the differential operator of the form

$$
B^{t, x}=\sum_{|r|=2 m} a_{r}(t, x) \frac{\partial^{|r|}}{\partial x_{1}^{r_{1}} \cdots \partial x_{n}^{r_{n}}}
$$

acting on functions defined on the space $\mathbb{R}^{n}$, satisfies the inequalities

$$
0<M_{1}|\xi|^{2 m} \leq(-1)^{m} B^{t, x}(\xi) \leq M_{2}|\xi|^{2 m}<\infty
$$

for $\xi \neq 0$. Problem (45) has a unique smooth solution. This allows us to reduce problem (45) to the abstract Cauchy problem (2) in a Banach space $E=C^{\mu}\left(\mathbb{R}^{n}\right)$ of all continuous bounded functions defined on $\mathbb{R}^{n}$ satisfying the Hölder condition with the indicator $\mu \in$ $(0,1)$ with a strongly positive operator $A^{t, x}=B^{t, x}+\delta I$ defined by $(52)$ (see $\left.[57,58]\right)$.

Theorem 3.1 For the solution of boundary problem (45), the following estimates are satisfied:

$$
\begin{aligned}
& \left\|D_{t}^{\frac{1}{2}} v\right\|_{C\left(C^{\mu}\left(\mathbb{R}^{n}\right)\right)} \leq M(\mu)\|f\|_{C\left(C^{\mu}\left(\mathbb{R}^{n}\right)\right)}, \quad 0 \leq \mu \leq 1, \\
& \left\|v_{t}\right\|_{C\left(C^{\mu+2 m \alpha}\left(\mathbb{R}^{n}\right)\right)} \leq M(\alpha, \mu)\|f\|_{C\left(C^{\mu+2 m \alpha}\left(\mathbb{R}^{n}\right)\right)}, \quad 0<2 m \alpha+\mu<1 .
\end{aligned}
$$

The proof of Theorem 3.1 is based on the abstract Theorems 2.1, 2.3, 2.4 and the coercivity inequality for an elliptic operator $A^{t, x}$ in $C^{\mu}\left(\mathbb{R}^{n}\right)$ and on the following theorem on the structure of the fractional spaces $E_{\alpha}\left(C^{\mu}\left(\mathbb{R}^{n}\right), A^{t, x}\right)$.

Theorem 3.2 $E_{\alpha}\left(C^{\mu}\left(\mathbb{R}^{n}\right), A^{t, x}\right)=C^{2 m \alpha+\mu}\left(\mathbb{R}^{n}\right)$ for all $0<2 m \alpha+\mu<1$ and $0 \leq t \leq 1$ [59].

Second, we consider the mixed boundary value problem for the fractional parabolic equation

$$
\left\{\begin{array}{l}
\frac{\partial v(t, x)}{\partial t}+D_{t}^{\frac{1}{2}} v(t, x)-a(t, x) \frac{\partial^{2} v(t, x)}{\partial x^{2}}+\sigma v(t, x)=f(t, x), \quad 0<t<1,0<x<1 \\
v(0, x)=0, \quad 0 \leq x \leq 1 \\
u(t, 0)=u(t, 1), \quad u_{x}(t, 0)=u_{x}(t, 1), \quad 0 \leq t \leq 1
\end{array}\right.
$$


where $a(t, x)$ and $f(t, x)$ are given sufficiently smooth functions and $a(t, x) \geq a>0$. Here, $\sigma$ is a sufficiently large positive constant.

We introduce the Banach spaces $C^{\beta}[0,1](0<\beta<1)$ of all continuous functions $\varphi(x)$ satisfying the Hölder condition for which the following norms are finite:

$$
\|\varphi\|_{C^{\beta}[0,1]}=\|\varphi\|_{C[0,1]}+\sup _{0 \leq x<x+\tau \leq 1} \frac{|\varphi(x+\tau)-\varphi(x)|}{\tau^{\beta}},
$$

where $C[0,1]$ is the space of all continuous functions $\varphi(x)$ defined on $[0,1]$ with the usual norm

$$
\|\varphi\|_{C[0,1]}=\max _{0 \leq x \leq 1}|\varphi(x)| .
$$

It is known that the differential expression [60]

$$
A^{t, x} v=-a(t, x) v^{\prime \prime}(t, x)+\sigma v(t, x)
$$

defines a positive operator $A^{t, x}$ acting in $C^{\beta}[0,1]$ with the domain $C^{\beta+2}[0,1]$ and satisfying the conditions $v(t, 0)=v(t, 1), v_{x}(t, 0)=v_{x}(t, 1)$. Therefore, we can replace the mixed problem (56) by the abstract boundary value problem (2). Using the results of Theorems 2.1, 2.3, 2.4, we can obtain the following theorem.

Theorem 3.3 For the solution of mixed problem (56), the following estimates are valid:

$$
\begin{aligned}
& \left\|D_{t}^{\frac{1}{2}} v\right\|_{C\left(C^{\mu}[0,1]\right)} \leq M(\mu)\|f\|_{C\left(C^{\mu}[0,1]\right)}, \quad 0 \leq \mu \leq 1, \\
& \left\|v_{t}\right\|_{C\left(C^{\mu+2 m \alpha}[0,1]\right)} \leq M(\alpha, \mu)\|f\|_{C\left(C^{\mu+2 m \alpha}[0,1]\right)}, \quad 0<2 m \alpha+\mu<1 .
\end{aligned}
$$

The proof of Theorem 3.3 is based on abstract Theorems 2.1, 2.3, 2.4 and on the following theorem on the structure of the fractional spaces $E_{\alpha}\left(C[0,1], A^{t, x}\right)$.

Theorem 3.4 $E_{\alpha}\left(C[0,1], A^{t, x}\right)=C^{2 \alpha}[0,1]$ for all $0<\alpha<\frac{1}{2}, 0 \leq t \leq 1[60]$.

\section{The well-posedness of problem (3)}

Let us first obtain the representation for the solution of problem (3). It is clear that the first order of accuracy difference scheme

$$
\tau^{-1}\left(u_{k}-u_{k-1}\right)+A_{k} u_{k}=F_{k}, \quad 1 \leq k \leq N, \quad N \tau=1, \quad u_{0}=0
$$

has a solution and the following formula holds:

$$
u_{k}=\sum_{s=1}^{k} u_{\tau}(k, s) F_{s} \tau, \quad 1 \leq k \leq N
$$

where

$$
u_{\tau}(k, j)= \begin{cases}R_{k} \cdots R_{j+1}, & k>j, \\ I, & k=j .\end{cases}
$$


Here $R_{k}=\left(I+\tau A_{k}\right)^{-1}$. Denote that

$$
D_{\tau}^{\frac{1}{2}} u_{k}=\frac{1}{\sqrt{\pi}} \sum_{m=1}^{k} \frac{\Gamma\left(k-m+\frac{1}{2}\right)}{(k-m) !} \frac{u_{m}-u_{m-1}}{\tau^{\frac{1}{2}}} .
$$

Applying the formula $F_{k}=f_{k}-D_{\tau}^{\frac{1}{2}} u_{k}$, we get

$$
u_{k}=-\sum_{s=1}^{k} u_{\tau}(k, s) D_{\tau}^{\frac{1}{2}} u_{s} \tau+\sum_{s=1}^{k} u_{\tau}(k, s) f_{s} \tau, \quad 1 \leq k \leq N
$$

So, formula (66) gives the representation for the solution of problem (3).

Let $F_{\tau}(E)$ be the linear space of mesh functions $\varphi^{\tau}=\left\{\varphi_{k}\right\}_{1}^{N}$ with values in the Banach space $E$. Next on $F_{\tau}(E)$ we introduce the Banach space $C_{\tau}(E)=C\left([0,1]_{\tau}, E\right)$ with the norm

$$
\left\|\varphi^{\tau}\right\|_{C_{\tau}(E)}=\max _{1 \leq k \leq N}\left\|\varphi_{k}\right\|_{E} .
$$

Theorem 4.1 Let $A(t)$ be a strongly positive operator in a Banach space E. Then for the solution $u^{\tau}=\left\{u_{k}\right\}_{1}^{N}$ in $C_{\tau}(E)$ of initial value problem (3), the stability inequality

$$
\left\|\left\{D_{\tau}^{\frac{1}{2}} u_{k}\right\}_{1}^{N}\right\|_{C_{\tau}(E)}+\left\|\left\{\tau^{-1}\left(u_{k}-u_{k-1}\right)+A_{k} u_{k}\right\}_{1}^{N}\right\|_{C_{\tau}(E)} \leq M\left\|f^{\tau}\right\|_{C_{\tau}(E)}
$$

holds.

Proof Using formula (66), we get

$$
\tau^{-1}\left(u_{k}-u_{k-1}\right)=-D_{\tau}^{\frac{1}{2}} u_{k}+\sum_{s=1}^{k} A_{k} u_{\tau}(k, s) D_{\tau}^{\frac{1}{2}} u_{s} \tau+f_{k}-\sum_{s=1}^{k} A_{k} u_{\tau}(k, s) f_{s} \tau
$$

Applying formulas (69) and (65), we obtain

$$
\begin{aligned}
D_{\tau}^{\frac{1}{2}} u_{k}= & \frac{1}{\sqrt{\pi}} \sum_{m=1}^{k} \frac{\Gamma\left(k-m+\frac{1}{2}\right)}{(k-m) !} \tau^{\frac{1}{2}}\left[-D_{\tau}^{\frac{1}{2}} u_{m}+f_{m}\right] \\
& +\frac{1}{\sqrt{\pi}} \sum_{m=1}^{k} \frac{\Gamma\left(k-m+\frac{1}{2}\right)}{(k-m) !}\left[\sum_{s=1}^{m} A_{k} u_{\tau}(k, s) D_{\tau}^{\frac{1}{2}} u_{s} \tau^{\frac{3}{2}}-\sum_{s=1}^{m} A_{k} u_{\tau}(k, s) f_{s} \tau^{\frac{3}{2}}\right] \\
= & \frac{1}{\sqrt{\pi}} \sum_{m=1}^{k} \frac{\Gamma\left(k-m+\frac{1}{2}\right)}{(k-m) !} \tau^{\frac{1}{2}}\left[-D_{\tau}^{\frac{1}{2}} u_{m}+f_{m}\right] \\
& +\frac{1}{\sqrt{\pi}} \sum_{s=1}^{k} \sum_{m=s}^{k} \frac{\Gamma\left(k-m+\frac{1}{2}\right)}{(k-m) !} A_{m} u_{\tau}(m, s) D_{\tau}^{\frac{1}{2}} u_{s} \tau^{\frac{3}{2}} \\
& -\frac{1}{\sqrt{\pi}} \sum_{s=1}^{k} \sum_{m=s}^{k} \frac{\Gamma\left(k-m+\frac{1}{2}\right)}{(k-m) !} A_{m} u_{\tau}(m, s) f_{s} \tau^{\frac{3}{2}} .
\end{aligned}
$$

Let us first obtain the estimate

$$
\left\|\frac{1}{\sqrt{\pi}} \sum_{m=s}^{k} \frac{\Gamma\left(k-m+\frac{1}{2}\right)}{(k-m) !} A_{m} u_{\tau}(m, s) \tau^{\frac{1}{2}}\right\|_{E \rightarrow E} \leq \frac{M}{\sqrt{(k-s) \tau}}
$$


for any $1 \leq s<k \leq N$. We have that

$$
\begin{aligned}
& \frac{1}{\sqrt{\pi}} \sum_{m=s}^{k} \frac{\Gamma\left(k-m+\frac{1}{2}\right)}{(k-m) !} A_{m} u_{\tau}(m, s) \tau^{\frac{1}{2}} \\
& =\frac{1}{\sqrt{\pi}} \sum_{m=\left[\frac{s+k}{2}\right]}^{k} \frac{\Gamma\left(k-m+\frac{1}{2}\right)}{(k-m) !} A_{m} u_{\tau}(m, s) \tau^{\frac{1}{2}} \\
& \quad+\frac{1}{\sqrt{\pi}} \sum_{m=s}^{\left[\frac{s+k}{2}\right]-1} \frac{\Gamma\left(k-m+\frac{1}{2}\right)}{(k-m) !} A_{m} u_{\tau}(m, s) \tau^{\frac{1}{2}}=J_{1}+J_{2} .
\end{aligned}
$$

Using estimates

$$
\left\|A_{k} u_{\tau}(k, s)\right\|_{E \rightarrow E} \leq \frac{M}{(k-s+1) \tau}, \quad\left\|u_{\tau}(k, s)\right\|_{E \rightarrow E} \leq M, \quad 1 \leq k \leq N
$$

and the following elementary inequality:

$$
\frac{\Gamma\left(k-m+\frac{1}{2}\right)}{(k-m) !} \leq \frac{1}{\sqrt{k-m}}, \quad 0 \leq m<k
$$

we obtain

$$
\begin{aligned}
\left\|J_{1}\right\|_{E \rightarrow E} & \leq \frac{1}{\sqrt{\pi}} \sum_{m=\left[\frac{s+k}{2}\right]}^{k} \frac{\Gamma\left(k-m+\frac{1}{2}\right)}{(k-m) !}\left\|A_{m} u_{\tau}(m, s)\right\|_{E \rightarrow E} \tau^{\frac{1}{2}} \\
& \leq M \frac{1}{\sqrt{\pi}} \sum_{m=\left[\frac{s+k}{2}\right]}^{k} \frac{\Gamma\left(k-m+\frac{1}{2}\right)}{(k-m) !} \frac{1}{(m-s+1) \tau} \tau^{\frac{1}{2}} \\
& \leq \frac{2 M}{(k-s) \tau} \frac{1}{\sqrt{\pi}} \sum_{m=\left[\frac{s+k}{2}\right]}^{k} \frac{\tau}{\sqrt{(k-m) \tau}} \leq \frac{M_{1}}{\sqrt{(k-s) \tau}} .
\end{aligned}
$$

Now, we will estimate $J_{2}$. We have that

$$
\begin{aligned}
J_{2}= & \frac{1}{\sqrt{\pi}} \frac{\Gamma\left(k-s+\frac{1}{2}\right)}{(k-s) !} \tau^{-\frac{1}{2}}-\frac{1}{\sqrt{\pi}} \frac{\Gamma\left(k-\left[\frac{s+k}{2}\right]+\frac{3}{2}\right)}{\left(k-\left[\frac{s+k}{2}\right]+1\right) !} u_{\tau}\left(\left[\frac{s+k}{2}\right], s\right) \tau^{-\frac{1}{2}} \\
& +\frac{1}{\sqrt{\pi}} \sum_{m=s+1}^{\left[\frac{s+k}{2}\right]-1}\left[\frac{\Gamma\left(k-m+\frac{1}{2}\right)}{(k-m) !}-\frac{\Gamma\left(k-m+\frac{3}{2}\right)}{(k-m+1) !}\right] u_{\tau}(m-1, s) \tau^{-\frac{1}{2}} .
\end{aligned}
$$

Applying estimates (73) and (74), we get

$$
\begin{aligned}
\left\|J_{2}\right\|_{E \rightarrow E} \leq & \frac{1}{\sqrt{\pi}} \frac{1}{\sqrt{(k-s) \tau}}+\frac{1}{\sqrt{\pi}}\left\|u_{\tau}\left(\left[\frac{s+k}{2}\right], s\right)\right\|_{E \rightarrow E} \frac{1}{\sqrt{\left(k-\left[\frac{s+k}{2}\right]+1\right) \tau}} \\
& +\frac{1}{\sqrt{\pi}} \sum_{m=s+1}^{\left[\frac{s+k}{2}\right]-1}\left|\frac{\Gamma\left(k-m+\frac{1}{2}\right)}{(k-m) !}-\frac{\Gamma\left(k-m+\frac{3}{2}\right)}{(k-m+1) !}\right|\left\|u_{\tau}(m-1, s)\right\|_{E \rightarrow E} \tau^{-\frac{1}{2}}
\end{aligned}
$$




$$
\begin{aligned}
\leq & \frac{1}{\sqrt{\pi}} \frac{1}{\sqrt{(k-s) \tau}}+\frac{1}{\sqrt{\pi}} M \frac{\sqrt{2}}{\sqrt{(k-s) \tau}} \\
& +M \frac{\frac{1}{2}}{\sqrt{\pi}} \sum_{m=s+1}^{\left[\frac{s+k}{2}\right]-1} \frac{\tau}{(k-m+1) \tau \sqrt{(k-m) \tau}} \leq \frac{M_{2}}{\sqrt{(k-s) \tau}} .
\end{aligned}
$$

Estimate (71) follows from estimates (75) and (80).

Now, let us first estimate $z_{k}=\left\|D_{\tau}^{\frac{1}{2}} u_{k}\right\|_{E}$. Applying the triangle inequality and estimate (71), we get

$$
\begin{aligned}
z_{k} \leq & \frac{1}{\sqrt{\pi}} \sum_{m=1}^{k} \frac{\Gamma\left(k-m+\frac{1}{2}\right)}{(k-m) !} \tau^{\frac{1}{2}}\left[z_{m}+\left\|f_{m}\right\|_{E}\right] \\
& +\frac{1}{\sqrt{\pi}} \sum_{s=1}^{k}\left\|\sum_{m=s}^{k} \frac{\Gamma\left(k-m+\frac{1}{2}\right)}{(k-m) !} A_{m} u_{\tau}(m, s)\right\|_{E \rightarrow E} z_{s} \tau^{\frac{3}{2}} \\
& +\frac{1}{\sqrt{\pi}} \sum_{s=1}^{k}\left\|\sum_{m=s}^{k} \frac{\Gamma\left(k-m+\frac{1}{2}\right)}{(k-m) !} A_{m} u_{\tau}(m, s)\right\|_{E \rightarrow E}\left\|f_{s}\right\|_{E} \tau^{\tau^{\frac{3}{2}}} \\
\leq & M_{3} \sum_{s=1}^{k-1} \frac{1}{\sqrt{(k-s) \tau}} \tau\left[z_{s}+\left\|f_{s}\right\|_{E}\right]+M_{4}\left[z_{k}+\left\|f_{k}\right\|_{E}\right] \tau^{\frac{1}{2}}
\end{aligned}
$$

for any $k=1, \ldots, N$. Applying the above inequality and the difference analogue of the integral inequality, we obtain

$$
\left\|\left\{D_{\tau}^{\frac{1}{2}} u_{k}\right\}_{1}^{N}\right\|_{C_{\tau}(E)} \leq M\left\|\left\{f_{k}\right\}_{1}^{N}\right\|_{C_{\tau}(E)} .
$$

Using the triangle inequality and equation (3), we get

$$
\begin{aligned}
& \left\|\left\{\tau^{-1}\left(u_{k}-u_{k-1}\right)+A_{k} u_{k}\right\}_{1}^{N}\right\|_{C_{\tau}(E)} \\
& \quad \leq\left[\left\|\left\{f_{k}\right\}_{1}^{N}\right\|_{C_{\tau}(E)}+\left\|\left\{D_{\tau}^{\frac{1}{2}} u_{k}\right\}_{1}^{N}\right\|_{C_{\tau}(E)}\right] \\
& \quad \leq M_{1}\left\|f^{\tau}\right\|_{C_{\tau}(E)} .
\end{aligned}
$$

Estimate (68) follows from estimates (88) and (89). Theorem 4.1 is proved.

With the help of $A(t)$, we introduce the fractional spaces $E_{\alpha}^{\prime}=E_{\alpha}^{\prime}(E, A(t)), 0<\alpha<1$, consisting of all $v \in E$ for which the following norms are finite:

$$
\|v\|_{E_{\alpha}^{\prime}}=\sup _{\lambda>0} \lambda^{\alpha}\left\|A(t)(\lambda+A(t))^{-1} v\right\|_{E^{*}}
$$

From (73) it follows that

Theorem 4.2 $E_{\alpha}^{\prime}(E, A(t))=E_{\alpha}^{\prime}(E, A(0))$ for all $0<\alpha<1$ and $0 \leq t \leq 1$.

Problem (3) is not well posed in $C_{\tau}(E)$ for arbitrary $E$. It turns out that a Banach space $E$ can be restricted to a Banach space $E^{\prime}$ in such a manner that the restricted problem (3) in $E^{\prime}$ will be well posed in $C\left(E^{\prime}\right)$. The role of $E^{\prime}$ will be played here by the fractional spaces $E_{\alpha}=E_{\alpha}(A(t), E)(0<\alpha<1)$. 
Theorem 4.3 Suppose that assumptions (6) and (7) hold and $0<\alpha \leq \varepsilon<1$. Then for the solution $u^{\tau}=\left\{u_{k}\right\}_{1}^{N}$ in $C_{\tau}\left(E_{\alpha}^{\prime}\right)$ of initial value problem (3), the coercive stability inequality

$$
\begin{aligned}
& \left\|\left\{\tau^{-1}\left(u_{k}-u_{k-1}\right)\right\}_{1}^{N}\right\|_{C_{\tau}\left(E_{\alpha}^{\prime}\right)}+\left\|\left\{A_{k} u_{k}\right\}_{1}^{N}\right\|_{C_{\tau}\left(E_{\alpha}^{\prime}\right)} \\
& \quad \leq M \alpha^{-1}(1-\alpha)^{-1}\left\|f^{\tau}\right\|_{C_{\tau}\left(E_{\alpha}^{\prime}\right)}
\end{aligned}
$$

holds.

Proof By Theorem 4.1,

$$
\left\|\left\{D_{\tau}^{\frac{1}{2}} u_{k}\right\}_{1}^{N}\right\|_{C_{\tau}\left(E_{\alpha}^{\prime}\right)} \leq M\left\|f^{\tau}\right\|_{C_{\tau}\left(E_{\alpha}^{\prime}\right)}
$$

for the solution of initial value problem (3). The proof of the estimate

$$
\left\|\left\{A_{k} u_{k}\right\}_{1}^{N}\right\|_{C_{\tau}\left(E_{\alpha}^{\prime}\right)} \leq M \alpha^{-1}(1-\alpha)^{-1}\left\|f^{\tau}\right\|_{C_{\tau}\left(E_{\alpha}^{\prime}\right)}
$$

for the solution of initial value problem (3) is based on estimate (92) and the following estimates [51]:

$$
\begin{aligned}
& \max _{1 \leq k \leq N}\left\|\sum_{s=1}^{k} A_{k} u_{\tau}(k, s) f_{s} \tau\right\|_{E_{\alpha}^{\prime}} \leq M \alpha^{-1}(1-\alpha)^{-1}\left\|f^{\tau}\right\|_{C\left(E_{\alpha}^{\prime}\right)}, \\
& \max _{1 \leq k \leq N}\left\|\sum_{s=1}^{k} A_{k} u_{\tau}(k, s) D_{\tau}^{\frac{1}{2}} u_{s} \tau\right\|_{E_{\alpha}^{\prime}} \leq M \alpha^{-1}(1-\alpha)^{-1}\left\|\left\{D_{\tau}^{\frac{1}{2}} u_{k}\right\}_{1}^{N}\right\|_{C\left(E_{\alpha}^{\prime}\right)} .
\end{aligned}
$$

Using the triangle inequality and equation (3), we get

$$
\begin{aligned}
& \left\|\left\{\tau^{-1}\left(u_{k}-u_{k-1}\right)\right\}_{1}^{N}\right\|_{C_{\tau}\left(E_{\alpha}^{\prime}\right)} \\
& \quad \leq\left[\left\|f^{\tau}\right\|_{C\left(E_{\alpha}^{\prime}\right)}+\left\|\left\{A_{k} u_{k}\right\}_{1}^{N}\right\|_{C_{\tau}\left(E_{\alpha}^{\prime}\right)}+\left\|\left\{D_{\tau}^{\frac{1}{2}} u\right\}_{1}^{N}\right\|_{C\left(E_{\alpha}^{\prime}\right)}\right] \\
& \quad \leq M_{1} \alpha^{-1}(1-\alpha)^{-1}\left\|f^{\tau}\right\|_{C\left(E_{\alpha}^{\prime}\right)} .
\end{aligned}
$$

Estimate (91) follows from estimates (93) and (96). Theorem 4.3 is proved.

Let us give, without proof, the following result.

Theorem 4.4 Suppose that assumptions (6) and (43) hold. Then for the solution $u^{\tau}=$ $\left\{u_{k}\right\}_{1}^{N}$ in $C_{\tau}\left(E_{\alpha}^{\prime}\right)$ of initial value problem (3), the coercive stability inequality

$$
\begin{aligned}
& \left\|\left\{\tau^{-1}\left(u_{k}-u_{k-1}\right)\right\}_{1}^{N}\right\|_{C_{\tau}\left(E_{\alpha}^{\prime}\right)}+\left\|\left\{A_{k} u_{k}\right\}_{1}^{N}\right\|_{C_{\tau}\left(E_{\alpha}^{\prime}\right)} \\
& \quad \leq M \alpha^{-1}(1-\alpha)^{-1}\left\|f^{\tau}\right\|_{C_{\tau}\left(E_{\alpha}^{\prime}\right)}
\end{aligned}
$$

holds.

Note that by passing to the limit for $\tau \rightarrow 0$, one can recover Theorems 2.1-2.3 and 2.4. 


\section{Applications}

Now, we consider the applications of Theorems 4.1, 4.3 and 4.4.

First, initial value problem (45) is considered. The discretization of problem (45) is carried out in two steps. In the first step, the grid space $\mathbb{R}_{h}^{n}\left(0<h \leq h_{0}\right)$ is defined as the set of all points of the Euclidean space $\mathbb{R}^{n}$ whose coordinates are given by

$$
x_{k}=s_{k} h, \quad s_{k}=0, \pm 1, \pm 2, \ldots, k=1, \ldots, n .
$$

The difference operator $A_{h}^{t, x}=B_{h}^{t, x}+\sigma I_{h}$ is assigned to the differential operator $A^{x}=B^{x}+\sigma I$, defined by (52). The operator

$$
B_{h}^{t, x}=h^{-2 m} \sum_{2 m \leq|s| \leq S} b_{s}^{t, x} \Delta_{1-}^{s_{1}} \Delta_{1+}^{s_{2}} \cdots \Delta_{n-}^{s_{2 n-1}} \Delta_{n+}^{s_{2 n}}
$$

acts on functions defined on the entire space $\mathbb{R}_{h}^{n}$. Here $s \in \mathbb{R}^{2 n}$ is a vector with nonnegative integer coordinates,

$$
\Delta_{k \pm} f^{h}(x)= \pm\left(f^{h}\left(x \pm e_{k} h\right)-f^{h}(x)\right)
$$

where $e_{k}$ is the unit vector of the axis $x_{k}$.

An infinitely differentiable function $\varphi(x)$ of the continuous argument $x \in \mathbb{R}^{n}$ that is continuous and bounded together with all its derivatives is said to be smooth. We say that the difference operator $A_{h}^{t, x}$ is a $\lambda$ th order $(\lambda>0)$ approximation of the differential operator $A^{t, x}$ if the inequality

$$
\sup _{x \in \mathbb{R}_{h}^{n}}\left|A_{h}^{t, x} \varphi(x)-A^{t, x} \varphi(x)\right| \leq M(\varphi) h^{\lambda}
$$

holds for any smooth function $\varphi(x)$. The coefficients $b_{s}^{t, x}$ are chosen in such a way that the operator $A_{h}^{t, x}$ approximates in a specified way the operator $A^{t, x}$. It will be assumed that the operator $A_{h}^{t, x}$ approximates the differential operator $A^{t, x}$ with any prescribed order $[57,58]$.

The function $A^{t, x}(\xi h, h)$ is obtained by replacing the operator $\Delta_{k \pm}$ in the right-hand side of equality (99) with the expression $\pm\left(\exp \left\{ \pm i \xi_{k} h\right\}-1\right)$, respectively, and is called the symbol of the difference operator $B_{h}^{t, x}$.

It will be assumed that for $\left|\xi_{k} h\right| \leq \pi$ and fixed $x$, the symbol $A^{t, x}(\xi h, h)$ of the operator $B_{h}^{t, x}=A_{h}^{t, x}-\sigma I_{h}$ satisfies the inequalities

$$
(-1)^{m} A^{t, x}(\xi h, h) \geq M|\xi|^{2 m}, \quad\left|\arg A^{t, x}(\xi h, h)\right| \leq \phi<\phi_{0} \leq \frac{\pi}{2} .
$$

Suppose that the coefficient $b_{s}^{x}$ of the operator $B_{h}^{t, x}=A_{h}^{t, x}-\sigma I_{h}$ is bounded and satisfies the inequalities

$$
\left|b_{s}^{t, x+e_{k} h}-b_{s}^{t, x}\right| \leq M h^{\epsilon}, \quad x \in \mathbb{R}_{h}^{n}, \epsilon \in(0,1] .
$$


With the help of $A_{h}^{t, x}$, we arrive at the nonlocal boundary value problem

$$
\left\{\begin{array}{l}
\frac{d v^{h}(t, x)}{d t}+D_{t}^{\frac{1}{2}} v^{h}(t, x)+A_{h}^{t, x} v^{h}(t, x)=f^{h}(t, x), \quad 0<t<1, x \in \mathbb{R}_{h}^{n}, \\
v^{h}(0, x)=0, \quad x \in \mathbb{R}_{h}^{n}
\end{array}\right.
$$

for an infinite system of ordinary differential equations.

In the second step, problem (104) is replaced by the difference scheme

$$
\left\{\begin{array}{l}
\frac{u_{k}^{h}(x)-u_{k-1}^{h}(x)}{\tau}+\frac{1}{\sqrt{\pi}} \sum_{m=1}^{k} \frac{\Gamma\left(k-m+\frac{1}{2}\right)}{(k-m) !} \frac{u_{m}^{h}-u_{m-1}^{h}}{\tau^{\frac{1}{2}}}+A_{h}^{k, x} u_{k}^{h}=f_{k}^{h}(x), \\
f_{k}^{h}(x)=f^{h}\left(t_{k}, x\right), \quad t_{k}=k \tau, \quad 1 \leq k \leq N-1, \quad N \tau=1, \quad x \in \mathbb{R}_{h}^{n}, \\
u_{0}^{h}(x)=0, \quad x \in \mathbb{R}_{h}^{n} .
\end{array}\right.
$$

Based on the number of corollaries of the abstract theorems given in the above, to formulate the result, one needs to introduce the spaces $C_{h}=C\left(\mathbb{R}_{h}^{n}\right)$ and $C_{h}^{\beta}=C^{\beta}\left(\mathbb{R}_{h}^{n}\right)$ of all bounded grid functions $u^{h}(x)$ defined on $\mathbb{R}_{h}^{n}$, equipped with the norms

$$
\begin{aligned}
& \left\|u^{h}\right\|_{C_{h}}=\sup _{x \in \mathbb{R}_{h}^{n}}\left|u^{h}(x)\right|, \\
& \left\|u^{h}\right\|_{C_{h}^{\beta}}=\sup _{x \in \mathbb{R}_{h}^{n}}\left|u^{h}(x)\right|+\sup _{x, y \in \mathbb{R}_{h}^{n}} \frac{\left|u^{h}(x)-u^{h}(x+y)\right|}{|y|^{\beta}} .
\end{aligned}
$$

Theorem 5.1 Suppose that assumptions (102) and (103) for the operator $A_{h}^{k, x}$ hold. Then, the solutions of difference scheme (105) satisfy the following stability estimates:

$$
\begin{gathered}
\max _{1 \leq k \leq N}\left\|D_{\tau}^{\frac{1}{2}} u_{k}^{h}\right\|_{C_{h}^{\mu}} \leq M_{1}(\mu) \max _{1 \leq k \leq N}\left\|f_{k}^{h}\right\|_{C_{h}^{\mu}}, \quad 0 \leq \mu \leq 1, \\
\left\|\left\{\tau^{-1}\left(u_{k}^{h}-u_{k-1}^{h}\right)\right\}_{1}^{N}\right\|_{C_{\tau}\left(C_{h}^{\mu+2 m \alpha}\right)}+\left\|\left\{A_{k} u_{k}\right\}_{1}^{N}\right\|_{C_{\tau}\left(C_{h}^{\mu+2 m \alpha}\right)} \\
\leq M(\alpha, \mu) \max _{1 \leq k \leq N}\left\|f_{k}^{h}\right\|_{C_{h}^{\mu+2 m \alpha}}, \quad 0<2 m \alpha+\mu<1 .
\end{gathered}
$$

The proof of Theorem 5.1 is based on the abstract Theorems 4.1, 4.3, 4.4 and the strong positivity of the operator $A_{h}^{x}$ defined by (114) in $C_{h}^{\mu}$ and on the following two theorems on the coercivity inequality for the solution of the elliptic difference equation in $C_{h}^{\beta}$ and on the structure of the fractional space $E_{\alpha}^{\prime}\left(C_{h}, A_{h}^{x}\right)$.

Theorem 5.2 Suppose that assumptions (102) and (103) for the operator $A_{h}^{k, x}$ hold. Then for the solutions of the elliptic difference equation

$$
A_{h}^{k, x} u^{h}(x)=\omega^{h}(x), \quad x \in \mathbb{R}_{h}^{n},
$$

the estimates [54]

$$
\sum_{2 m \leq|s| \leq S} h^{-2 m}\left\|\Delta_{1-}^{s_{1}} \Delta_{1+}^{s_{2}} \cdots \Delta_{n-}^{s_{2 n-1}} \Delta_{n+}^{s_{2 n}} u^{h}\right\|_{C_{h}^{\beta}} \leq M(\sigma, \beta)\left\|\omega^{h}\right\|_{C_{h}^{\beta}}
$$

are valid. 
Theorem 5.3 Suppose that assumptions (102) and (103) for the operator $A_{h}^{k, x}$ hold. Then for any $0<\alpha<\frac{1}{2 m}$, the norms in the spaces $E_{\alpha}^{\prime}\left(C_{h}, A_{h}^{x}\right)$ and $C_{h}^{2 m \alpha}$ are equivalent uniformly in $h$ [51].

Second, we consider mixed boundary value problem (56). The discretization of problem (56) is carried out in two steps. In the first step, let us define the grid space

$$
[0,1]_{h}=\left\{x: x_{r}=r h, 0 \leq r \leq K, K h=1\right\} .
$$

We introduce the Banach space $C_{h}^{\beta}=C^{\beta}\left([0,1]_{h}\right)(0<\beta<1)$ of the grid functions $\varphi^{h}(x)=$ $\left\{\varphi_{r}\right\}_{1}^{K-1}$ defined on $[0,1]_{h}$, equipped with the norm

$$
\left\|\varphi^{h}\right\|_{C_{h}^{\beta}}=\left\|\varphi^{h}\right\|_{C_{h}}+\sup _{1 \leq k<k+\tau \leq K-1} \frac{\left|\varphi_{k+r}-\varphi_{k}\right|}{\tau^{\beta}}
$$

where $C_{h}=C\left([0,1]_{h}\right)$ is the space of the grid functions $\varphi^{h}(x)=\left\{\varphi_{r}\right\}_{1}^{K-1}$ defined on $[0,1]_{h}$, equipped with the norm

$$
\left\|\varphi^{h}\right\|_{C_{h}}=\max _{1 \leq k \leq K-1}\left|\varphi_{k}\right|
$$

To the differential operator $A$ generated by problem (56), we assign the difference operator $A_{h}^{x}$ by the formula

$$
A_{h}^{t, x} \varphi^{h}(x)=\left\{-\left(a(t, x) \varphi_{x}^{-}\right)_{x, r}+\delta \varphi_{r}\right\}_{1}^{K-1}
$$

acting in the space of grid functions $\varphi^{h}(x)=\left\{\varphi_{r}\right\}_{0}^{K}$ satisfying the conditions $\varphi_{0}=\varphi_{K}, \varphi_{1}-$ $\varphi_{0}=\varphi_{K}-\varphi_{K-1}$. With the help of $A_{h}^{x}$, we arrive at the initial boundary value problem

$$
\left\{\begin{array}{l}
\frac{d v^{h}(t, x)}{d t}+D_{t}^{\frac{1}{2}} v^{h}(t, x)+A_{h}^{t, x} v^{h}(t, x)=f^{h}(t, x), \quad 0<t<1, x \in[0,1]_{h}, \\
v^{h}(0, x)=0, \quad x \in[0,1]_{h}
\end{array}\right.
$$

for an infinite system of ordinary fractional differential equations. In the second step, we replace problem (115) by difference scheme (3)

$$
\begin{aligned}
& \frac{u_{k}^{h}(x)-u_{k-1}^{h}(x)}{\tau}+\frac{1}{\sqrt{\pi}} \sum_{m=1}^{k} \frac{\Gamma\left(k-m+\frac{1}{2}\right)}{(k-m) !} \frac{u_{m}^{h}(x)-u_{m-1}^{h}(x)}{\tau^{\frac{1}{2}}}+A_{h}^{k, x} u_{k}^{h}(x)=f_{k}^{h}(x), \\
& f_{k}^{h}(x)=\left\{f\left(t_{k}, x_{r}\right)\right\}_{1}^{K-1}, \\
& t_{k}=k \tau, \quad 1 \leq k \leq N-1, \quad N \tau=1 ; \quad u_{0}^{h}(x)=0, \quad x \in[0,1]_{h} .
\end{aligned}
$$

Theorem 5.4 Let $\tau$ and $h$ be sufficiently small numbers. Then, the solutions of difference scheme (116) satisfy the following stability estimates:

$$
\begin{aligned}
& \max _{1 \leq k \leq N}\left\|D_{\tau}^{\frac{1}{2}} u_{k}^{h}\right\|_{C_{h}^{\mu}} \leq M_{1}(\mu) \max _{1 \leq k \leq N}\left\|f_{k}^{h}\right\|_{C_{h}^{\mu}}, \quad 0 \leq \mu \leq 1, \\
& \left\|\left\{\tau^{-1}\left(u_{k}^{h}-u_{k-1}^{h}\right)\right\}_{1}^{N}\right\|_{C_{\tau}\left(C_{h}^{\mu+2 \alpha}\right)}+\left\|\left\{A_{k} u_{k}\right\}_{1}^{N}\right\|_{C_{\tau}\left(C_{h}^{\mu+2 \alpha}\right)} \\
& \leq M(\alpha, \mu) \max _{1 \leq k \leq N}\left\|f_{k}^{h}\right\|_{C_{h}^{\mu+2 \alpha}, \quad 0<2 \alpha+\mu<1 .}
\end{aligned}
$$


The proof of Theorem 5.4 is based on the abstract Theorems 4.1, 4.3, 4.4 and the strong positivity of the operator $A_{h}^{t, x}$ defined by (114) in $C_{h}^{\mu}$ and on the following theorem on the structure of the fractional space $E_{\alpha}^{\prime}\left(C_{h}, A_{h}^{t, x}\right)$.

\author{
Theorem 5.5 For any $0<\alpha<\frac{1}{2}$, the norms in the spaces $E_{\alpha}^{\prime}\left(C_{h}, A_{h}^{t, x}\right)$ and $C_{h}^{2 \alpha}$ are equiva- \\ lent uniformly in $h$ and $t \in[0,1][60]$.
}

\title{
Competing interests
}

The author declares that they have no competing interests.

\section{Acknowledgements}

The author would like to thank Prof. P. E. Sobolevskii for his helpful suggestions to the improvement of this paper.

Received: 2 October 2012 Accepted: 25 January 2013 Published: 18 February 2013

\section{References}

1. Podlubny, I: Fractional Differential Equations. Mathematics in Science and Engineering, vol. 198. Academic Press, San Diego (1999)

2. Samko, SG, Kilbas, AA, Marichev, Ol: Fractional Integrals and Derivatives. Gordon \& Breach, Yverdon (1993)

3. Kilbas, AA, Sristava, HM, Trujillo, JJ: Theory and Applications of Fractional Differential Equations, vol. 204. Elsevier, Amsterdam (2006)

4. El-Mesiry, AEM, El-Sayed, AMA, El-Saka, HAA: Numerical methods for multi-term fractional (arbitrary) orders differential equations. Appl. Math. Comput. 160(3), 683-699 (2005)

5. Diethelm, K: The Analysis of Fractional Differential Equations. Springer, Berlin (2010)

6. Pedas, A, Tamme, E: On the convergence of spline collocation methods for solving fractional differential equations. J. Comput. Appl. Math. 235(12), 3502-3514 (2011)

7. Diethelm, K, Ford, NJ: Multi-order fractional differential equations and their numerical solution. Appl. Math. Comput. 154(3), 621-640 (2004)

8. El-Sayed, AMA, Gaafar, FM: Fractional order differential equations with memory and fractional-order relaxation-oscillation model. Pure Math. Appl. 12(3), 296-310 (2001)

9. Basset, AB: On the descent of a sphere in a viscous liquid. Q. J. Math. 42, 369-381 (1910)

10. Mainardi, F: Fractional calculus: some basic problems in continuum and statistical mechanics. In: Carpinteri, A, Mainardi, F (eds.) Fractals and Fractional Calculus in Continuum Mechanics, pp. 291-348. Springer, New York (1997)

11. Momani, S, Al-Khaled, K: Numerical solutions for systems of fractional differential equations by the decomposition method. Appl. Math. Comput. 162(3), 1351-1365 (2005)

12. Bagley, RL, Torvik, PJ: On the appearance of the fractional derivative in the behavior of real materials. J. Appl. Mech. 51, 294-298 (1984)

13. Matignon, D: Stability results for fractional differential equations with applications to control processing. In: Computational Engineering in System Application, Lille, France, vol. 2 (1996)

14. Lavoie, JL, Osler, TJ, Tremblay, R: Fractional derivatives and special functions. SIAM Rev. 18(2), 240-268 (1976)

15. Tarasov, VE: Fractional derivative as fractional power of derivative. Int. J. Math. 18, 281-299 (2007)

16. Ashyralyev, A, Dal, F, Pinar, Z: On the numerical solution of fractional hyperbolic partial differential equations. Math. Probl. Eng. 2009, Article ID 730465 (2009)

17. Ashyralyev, A: A note on fractional derivatives and fractional powers of operators. J. Math. Anal. Appl. 357(1), 232-236 (2009)

18. Podlubny, I, El-Sayed, AMA: On two definitions of fractional calculus. Slovak Academy of Science, Institute of Experimental Physics (1996)

19. Yakar, A, Koksal, ME: Existence results for solutions of nonlinear fractional differential equations. Abstr. Appl. Anal. 2012, Article ID 267108 (2012)

20. De la Sen, M: Positivity and stability of the solutions of Caputo fractional linear time-invariant systems of any order with internal point delays. Abstr. Appl. Anal. 2011, Article ID 161246 (2011)

21. Yuan, C: Two positive solutions for $(n-1,1)$-type semipositone integral boundary value problems for coupled systems of nonlinear fractional differential equations. Commun. Nonlinear Sci. Numer. Simul. 17(2), $930-942$ (2012)

22. De la Sen, $M$, Agarwal, RP, Ibeas, A, et al.: On the existence of equilibrium points, boundedness, oscillating behavior and positivity of a SVEIRS epidemic model under constant and impulsive vaccination. Adv. Differ. Equ. 2011, Article ID 748608 (2011)

23. De la Sen, M: About robust stability of Caputo linear fractional dynamic systems with time delays through fixed point theory. Fixed Point Theory Appl. 2011, Article ID 867932 (2011)

24. Yuan, C: Multiple positive solutions for semipositone $(n, p)$-type boundary value problems of nonlinear fractional differential equations. Anal. Appl. 9(1), 97-112 (2011)

25. Yuan, C: Multiple positive solutions for $(n-1,1)$-type semipositone conjugate boundary value problems for coupled systems of nonlinear fractional differential equations. Electron. J. Qual. Theory Differ. Equ. 2011, 13 (2011)

26. Agarwal, RP, Belmekki, M, Benchohra, M: A survey on semilinear differential equations and inclusions involving Riemann-Liouville fractional derivative. Adv. Differ. Equ. 2009, Article ID 981728 (2009)

27. Agarwal, RP, de Andrade, B, Cuevas, C: On type of periodicity and ergodicity to a class of fractional order differential equations. Adv. Differ. Equ. 2010, Article ID 179750 (2010) 
28. Agarwal, RP, de Andrade, B, Cuevas, C: Weighted pseudo-almost periodic solutions of a class of semilinear fractional differential equations. Nonlinear Anal., Real World Appl. 11, 3532-3554 (2010)

29. Berdyshev, AS, Cabada, A, Karimov, ET: On a non-local boundary problem for a parabolic-hyperbolic equation involving a Riemann-Liouville fractional differential operator. Nonlinear Anal. 75(6), 3268-3273 (2011)

30. Ashyralyev, A, Amanov, D: Initial-boundary value problem for fractional partial differential equations of higher order. Abstr. Appl. Anal. 2012, Article ID 973102 (2012)

31. Ashyralyev, A, Sharifov, YA: Existence and uniqueness of solutions of the system of nonlinear fractional differential equations with nonlocal and integral boundary conditions. Abstr. Appl. Anal. 2012, Article ID 594802 (2012)

32. Ashyralyev, A, Hicdurmaz, B: A note on the fractional Schrödinger differential equations. Kybernetes 40(5-6), 736-750 (2011)

33. Ashyralyev, A, Dal, F, Pinar, Z: A note on the fractional hyperbolic differential and difference equations. Appl. Math. Comput. 217(9), 4654-4664 (2011)

34. Kirane, M, Laskri, Y: Nonexistence of global solutions to a hyperbolic equation with a space-time fractional damping Appl. Math. Comput. 167(2), 1304-1310 (2005)

35. Kirane, M, Laskri, Y, Tatar, N-e: Critical exponents of Fujita type for certain evolution equations and systems with spatio-temporal fractional derivatives. J. Math. Anal. Appl. 312(2), 488-501 (2005)

36. Kirane, M, Malik, SA: The profile of blowing-up solutions to a nonlinear system of fractional differential equations. Nonlinear Anal., Theory Methods Appl. 73(12), 3723-3736 (2010)

37. Araya, D, Lizama, C: Almost automorphic mild solutions to fractional differential equations. Nonlinear Anal. 69(11), 3692-3705 (2008)

38. N'Guerekata, GM: A Cauchy problem for some fractional abstract differential equation with nonlocal conditions. Nonlinear Anal. 70, 1873-1876 (2009)

39. Mophou, GM, N'Guerekata, GM: Mild solutions for semilinear fractional differential equations. Electron. J. Differ. Equ. 2009, $21(2009)$

40. Mophou, GM, N'Guerekata, GM: Existence of mild solution for some fractional differential equations with nonlocal conditions. Semigroup Forum 79(2), 315-322 (2009)

41. Lakshmikantham, V: Theory of fractional differential equations. Nonlinear Anal. 69(10), 3337-3343 (2008)

42. Lakshmikantham, V, Devi, JV: Theory of fractional differential equations in Banach spaces. Eur. J. Pure Appl. Math. 1, 38-45 (2008)

43. Lakshmikantham, $\mathrm{V}$, Vatsala, A: Theory of fractional differential inequalities and applications. Commun. Appl. Anal. 11(3-4), 395-402 (2007)

44. Clement, P, Guerre-Delabrire, S: On the regularity of abstract Cauchy problems and boundary value problems. Atti Accad. Naz. Lincei, Rend. Lincei, Mat. Appl. 9(4), 245-266 (1998)

45. Agarwal, RP, Bohner, M, Shakhmurov, VB: Maximal regular boundary value problems in Banach-valued weighted spaces. Bound. Value Probl. 1, 9-42 (2005)

46. Shakhmurov, VB: Coercive boundary value problems for regular degenerate differential-operator equations. J. Math. Anal. Appl. 292(2), 605-620 (2004)

47. Lunardi, A: Analytic semigroups and optimal regularity in parabolic problems. In: Operator Theory Advances and Applications. Birkhäuser, Basel (1995)

48. Ashyralyev, A, Hanalyev, A, Sobolevskii, PE: Coercive solvability of nonlocal boundary value problem for parabolic equations. Abstr. Appl. Anal. 6(1), 53-61 (2001)

49. Sobolevskii, PE: The coercive solvability of difference equations. Dokl. Akad. Nauk SSSR 201(5), 1063-1066 (1971) (Russian)

50. Sobolevskii, PE: Some properties of the solutions of differential equations in fractional spaces. In: Trudy Nauchn.-Issled. Inst. Mat. Voronezh. Gos. Univ. No., vol. 14, pp. 68-74 (1975) (Russian)

51. Ashyralyev, A, Sobolevskii, PE: New difference schemes for partial differential equations. In: Operator Theory: Advances and Applications, vol. 148. Birkhäuser, Basel (2004)

52. Ashyralyev, A: Well-posedness of the Basset problem in spaces of smooth functions. Appl. Math. Lett. 24, 1176-1180 (2011)

53. Ashyralyev, A, Cakir, Z: On the numerical solution of fractional parabolic partial differential equations. AlP Conf. Proc. 1389, 617-620 (2011)

54. Ashyralyev, A: Well-posedness of parabolic differential and difference equations with the fractional differential operator. Malays. J. Math. Sci. 6(S), 73-89 (2012)

55. Cakir, Z: Stability of difference schemes for fractional parabolic PDE with the Dirichlet-Neumann conditions. Abstr. Appl. Anal. 2012, Article ID 463746 (2012)

56. Ashyralyev, A, Cakir, Z: On the numerical solution of fractional parabolic partial differential equations with the Dirichlet condition. Discrete Dyn. Nat. Soc. 2012, Article ID 696179 (2012)

57. Smirnitskii, YA, Sobolevskii, PE: Positivity of multidimensional difference operators in the C-norm. Usp. Mat. Nauk 36(4), 202-203 (1981) (Russian)

58. Smirnitskii, YA: Fractional powers of elliptic difference operators. PhD thesis, Voronezh State University, Voronezh (1983) (Russian)

59. Ashyralyev, A, Sobolevskii, PE: Well-posedness of parabolic difference equations. In: Operator Theory Advances and Applications. Birkhäuser, Basel (1994)

60. Ashyralyev, A: Fractional spaces generated by the positive differential and difference operator in a Banach space. In: Taş, K, Tenreiro Machado, JA, Baleanu, D (eds.) Proceedings of the Conference 'Mathematical Methods and Engineering', pp. 13-22. Springer, Berlin (2007)

doi:10.1186/1687-2770-2013-31

Cite this article as: Ashyralyev: Well-posedness of fractional parabolic equations. Boundary Value Problems 2013 2013:31. 